\title{
Face to face with the suicidal
}

\section{Gethin Morgan, Carole Buckley \& Mike Nowers}

The clinical assessment and management of suicide risk depends primarily on face to face contact with the individual who presents the risk, and aims to predict behaviour in the very near future. Whether or not clinical intervention prevents suicide depends a great deal on the clinician's skill in reaching out to the individual patient. This poses a dilemma, because much of what has been written about predicting suicide has been based on averaged data concerning longterm outcome in large cohorts of patients.

Meeting the individual at risk presents its own challenges. The present article is based on the premise that clinical skills need to be evaluated systematically. This may not be possible at the level of the double-blind controlled trials so often demanded in the evidence-based approach; yet we surely ignore evaluation of basic clinical practice at our peril. The clinical skills involved deserve far more attention than they appear to receive in general; by scrupulously observing them the clinician's potential for preventing suicide must surely be enhanced.

In this paper we discuss the assessment and management of suicide risk as it is met in day to day clinical situations. Basic clinical skills are first identified, followed by discussion of the hazards which may be encountered, and the way in which these may vary according to particular clinical problems and situations. Throughout, we need to acknowledge that though suicidal ideation is encountered very commonly in clinical psychiatric practice, suicide itself is rare, and its prediction far from perfect. Failure to predict suicide should not be regarded as necessarily related to a poor standard of clinical care.

\section{Risk factors}

The socio-demographic and clinical risk factors which are traditionally cited in assessing suicide risk are shown in Box 1. These are correlates and not necessarily causes, and they encompass many false positives and false negatives (i.e. they are of low sensitivity and specificity). They are by no means infallible, and they should be used as a back-up to inform direct clinical assessment based on interview with each patient. Such risk factors can be no more than a rough guide, particularly suggesting caution when present in an otherwise reassuring clinical picture. They are better at

Box 1. Some suicide risk factors

Old age

Male gender

Divorced $>$ widowed $>$ single

Unemployed or retired

Living alone (socially isolated)

Physical illness, especially terminal illness or painful or debilitating illness

History of deliberate self-harm

Family history of affective (mood) disorder, alcohol dependency or suicide

Bereavement in childhood

Social classes I and V

Psychiatric and personality disorders

Gethin Morgan is Emeritus Professor of Mental Health at the University of Bristol (41 St Michael's Hill, Bristol BS2 8DZ). He has attempted over many years to evaluate clinical skills in the assessment and management of suicide. Carole Buckley is a principal in general practice in Almondsbury, near Bristol. Mike Nowers is a consultant in the psychiatry of old age at Frenchay NHS Trust in Bristol. He has long pursued a research programme into suicide prevention and the management of deliberate self-harm, with particular emphasis on the needs of the elderly. 
predicting long-term risk over months and years rather than in the immediate days ahead, and in males at least the age factor may be misleading because suicide is now just as common in young as in older men. All categories of psychiatric illness are associated with an increased risk of suicide; some such as depression, alcohol dependency, drug addiction and schizophrenia, lead the field.

Attempts have been made to refine the predictive value of diagnostic categories which have greater predictive risk (Table 1), but their reliability remains low. The validity of risk factors varies from one situation to another. For example, among psychiatric in-patients the numbers of suicides in females may exceed those in males, reflecting the overall preponderance of females in this population. Standardised risk questionnaires are subject to similar limitations, but they can be useful if regularly administered in monitoring changes in level of suicide risk, particularly for psychiatric in-patients.

\section{Assessment: basic clinical skills}

Given the major deficiencies in predictive value derived from traditional risk factors, we have to conclude that face to face clinical skills are of primary and paramount importance in our clinical approach to suicide risk.

\section{Suicidal state of mind}

In reaching out to the suicidal we need to have a picture in our own minds of the psychological processes which we are likely to encounter. Denial of suicidal ideation may disguise the true level of risk, though deliberate deception is probably far less common than true fluctuation in level of risk, often from one hour to the next. This may be related to ambivalence, or to chance encounter with stressful factors. Either way, the level of suicide risk should be monitored regularly and frequently if we are to be effective in suicide prevention.

\section{Clinical assessment}

Suicidal ideation is not a diagnosis in itself, but a symptom of other underlying psychopathology. Suicide risk can only be assessed effectively by full clinical evaluation, consisting of a thorough review of the previous history and present illness, assessment of mental state and then a diagnostic

\section{Table 1. Suicide and psychiatric disorders: high-risk factors within high-risk diagnoses}

\section{Diagnosis}

Depression
(lifetime risk of suicide $=15 \%$ )

Schizophrenia

(lifetime risk of suicide $=10 \%$ )

Alcohol addiction

(lifetime risk of suicide $=3.4 \%$ )

Risk factors

Male, older
Persistent insomnia
Previous act of deliberate self-harm
Self-neglect
Severe illness
Impaired memory
Agitation
Panic attacks
Male
Younger
Unemployed
Previous act of deliberate self-harm
Depressive episodes
Anorexia/weight loss
More serious illness
Recurrent relapse
Fear of deterioration, especially in those of high intellectual ability

Male (peak age 40-60 years)

High level of dependency

Long history of drinking

Disruption of major interpersonal relationships

Depressed mood

Poor physical health

Poor work record in previous four years 
formulation. In this, situational factors may be just as important as psychological mechanisms within the individual at risk. Some kind of psychiatric disorder is encountered in over $90 \%$ of suicides during the weeks and months immediately prior to their death and requires clinical evaluation in its own right.

It must be self-evident that a crucial component of risk assessment involves evaluation of suicidal ideation. Indeed, this may be the paramount skill required, in view of the difficulty in identifying any consistent clinical stereotype for suicidal individuals (Morgan \& Stanton, 1997). Yet very little attempt has been paid to the technique whereby suicidal ideation can be assessed effectively, even when everything has been done to establish rapport with the patient. Initial reluctance to share such painful ideas, especially with a stranger, is the rule.

A progressive focusing down on specific suicidal ideas can usefully begin with general issues (see Box 2). Acknowledgement of suicidal ideation is often associated with an emotional catharsis, probably as a sense of relief that attempts are being made to help. Much then still needs to be done in discussing the intensity of such ideas, their frequency, the degree of resistance to them or circumstances which may reduce or increase their severity. The risk to others is also relevant, for example in the case of depressive psychotic ideation which can include relatives and key other persons in ideas of futility. The whole process should not be an interrogation; each step should be approached using open-ended questions, at a speed that will vary from one patient to another. When there is a reluctance to engage in discussion fully, it may occasionally be necessary to use leading questions, because it is of the utmost importance to gain access to the patient's ideation if the assessment of risk is to be fully effective. Carried out with sensitivity, such an approach is acceptable because it is unlikely that the idea of suicide can be implanted in someone who is otherwise not at risk, or that the patient can be harmed in this way. Nevertheless, forceful challenge due to impatience on our part, for example, in the case of a patient who repeatedly attends the accident and emergency department and who is impulsive and irritable, might conceivably in certain circumstances provoke high-risk acting-out behaviour in response.

Real suicide risk is certainly consistent with a willingness to discuss suicidal ideation, even with presenting it without prompting. Sometimes acknowledged suicidal ideas have other meanings; in some they can be a way of influencing a life situation rather than an actual wish to destroy the self, though quite often both mechanisms may be present in the same patient. Interpretation of
Box 2. A sequence of questions useful in assessing suicide risk

The questions should ideally be openended. They are designed to help discover whether the patient:

Hopes that things will turn out well

Gets pleasure out of life

Feels hopeful from day to day

Feels able to face each day

Sees 'point in it all'

Ever despairs about things

Feels that it is impossible to face the next day

Feels life to be a burden

Wishes it would all end

Knows why he or she feels this way (e.g. wants to be with a dead person, life is bleak, morbid guilt)

Has thought of ending life (if so, how persistently?)

Has thoughts about the possible method of suicide (is the means readily available?)

Has ever acted on any suicidal thoughts or intentions

Feels able to resist any suicidal thoughts

The interviewer must decide:

The level of risk the person poses to self or others

Ability to give reassurance about safety (e.g. until next appointment)

Circumstances likely to make things worse

About how help can be made available at any time

meaning must depend upon the overall clinical context and consistency, as well as previous patterns of behaviour shown by the individual concerned. Such clinical judgement is always difficult; it is easier if the relationship with the patient is already well established, and great caution is needed in making such an evaluation on meeting a patient for the first time.

\section{Hazards of assessment}

Suicidal individuals exhibit a wide range of behaviours, ranging from depressive withdrawal to seemingly deliberate provocative flouting of rules, even overt anger or aggressive acts. It is unlikely 
that there is a distinctive clinical stereotype for suicidal individuals, such is the clinical overlap with others who do not commit suicide. Gross variation in distress and levels of despair, often from hour to hour, does not rule out significant risk. Particularly important is the recognition that suicide risk can temporarily subside on removal from relevant stress factors, for example, by admission to hospital which can lead to a false, temporary and fragile improvement, only to relapse easily whenever such adverse effects are encountered again. A minority of individuals may deny suicidal ideation and intent, which nevertheless remains serious; they may even appear calm and clinically improved. Such a paradoxical finding is sometimes related to relief, when a final decision has been made to commit suicide, that the agony of psychological pain and indecision has finally ended. Such a group is particularly difficult to evaluate, but the overall clinical picture is crucial, and any inconsistencies such as denial following a life-threatening episode of self-harm, should indicate that caution is required (see Box 3).

\section{Management of risk}

Once significant risk has been identified, it is necessary to monitor it regularly and match the level of care appropriately. For psychiatric in-patients most schemes involve a range from constant close contact (in most severe risk situations ensuring close physical proximity of staff) through monitoring every 15-20 minutes, to the 'known place' level

Box 3. Hazards which may mislead the assessment and management of suicide risk

Deliberate denial of suicidal ideas

Variability in degree of distress

Misleading clinical improvement (removal from stress factors, yet problems remain unresolved)

Anger, resentment (National Confidential Inquiry: $33 \%$ suicides have previous history of aggressive behaviour)

Uncooperative and 'difficult' behaviour

Malignant alienation

Assumption that patient is 'manipulating' with empty threats

Erroneous evaluation of suicidal ideations whereby the patient agrees to remain in an agreed locality (Williams \& Morgan, 1996). Rather than representing 'police-like' surveillance, this whole process should aim to provide a form of supportive observation which is welcomed by the patient. Listening to, and just being with in a concerned sympathetic way, are powerful strategies for reducing suicide risk; their value is generally underestimated. All personnel who have responsibility for patient care should be in no doubt about the scheme which applies in the unit where they work, and written details should be easily available for reference. Terminology should be explicit, otherwise variable interpretation from one hospital ward to another can pose a hazard: a commonly used term 'special' observation is one example of this, or alternatively depending merely on numerical indices of level rather than terms which have unambiguous explicit meaning. This approach of graded supportive observation does not deny that open good communication in a hospital ward goes a long way to improving its potential in preventing suicide. But this is insufficient in itself, and extra precautions are needed in the form of specific levels of care out of recognition that situations of high risk cannot always be assumed safe merely on the basis of a patient's reassurance. Critics of this approach sometimes regard it as an unjustified intrusive act of policing, when in reality it aims to be an alliance built on a contract between patients and staff. The step-like levels of care are arbitrary, but experience suggests that it is important to ensure a considerable gap between constant care and the next step, of 15-20 minutes supportive observation. That is, staff should be fully convinced that significant improvement has occurred before relaxing the constant level of support. It seems good practice to allow nursing staff discretion to increase the level of supportive observation if they are concerned, but on the other hand a reduction of care level is appropriately an agreed decision between both nursing and medical staff. It should also be recognised that suicide risk and aggressive behaviour may be associated, and the safety of staff should always be kept in mind, especially when close physical proximity is regarded necessary at high-risk levels.

Management of suicide risk in hospital wards is subject to a range of hazards (see Box 4). Any disruption of ward routine may increase risk. Our techniques for monitoring the way levels of risk fluctuate are far from perfect. Some in-patient suicides are able to leave the ward to kill themselves or they do so while on leave (Morgan \& Priest, 1991): this suggests that the degree of persisting risk is not always recognised sufficiently. Symptomatic improvement should not be in itself sufficient 
reassurance, and the full clinical situation, especially the persistence of adverse events or situations, should always be taken into account. Patients often improve temporarily on being distanced from their life difficulties, only to relapse quickly on facing them again, either on hospital leave or when they finally return home. This may be one explanation for the long-recognised high-risk period during the weeks following final discharge from hospital. Premature discharge of suicidal patients merely because of symptomatic improvement is a real risk at the present time when in-patient facilities are under such great demand. Great care is required whenever patients who are detained under the Mental Health Act are considered for home leave; in particular, every clinical unit should have a fully understood and explicit policy regarding Section 17 , whereby the written consent for leave must be obtained from the relevant responsible medical officer before leave is allowed. Restricted patients are also subject to extra constraints which need to be observed fully (Bannerjee et al, 1995).

The physical hazards in any ward environment are seemingly endless; for example, patients determined to kill themselves may do so by strangulation using pipes beneath ward basins, hanging from any curtain rail which has not been fitted with a release mechanism to ensure that it is not weight-bearing, by jumping from windows or

Box 4. Problems encountered in the management of in-patients at risk of suicide

Danger times

Soon after admission

Between staff shifts

Patient on leave (special care needed in the case of detained patients, Section 17 of the Mental Health Act)

Bank Holidays, staff leave and other disruptions of routine

Premature discharge

Follow-up period

Physical hazards in the hospital environment Poor communication between staff

Lack of clear code of practice in the care of suicidal patients

Failure to involve others in treatment process

Poor technique in assessing and monitoring risk

Misleading clinical improvement

Terminal progressive alienation of patient

Failure to gain admission staircases, setting fire to themselves in the kitchen, or hoarding tablets (either from prescribed medication or over-the-counter purchase). Effective clinical audit of adverse events and suicides among psychiatric in-patients should help to identify factors which are especially important in any particular clinical unit.

A further hazard concerns a high level of general disruption in a psychiatric ward. Depressive suicidal patients might tolerate an admission to such a situation on the first occasion, but they may be very reluctant to return later (should this become advisable), often much to their disadvantage should suicide risk be high.

Finally there is the problem of terminal malignant alienation which may complicate the management of suicidal patients (Morgan, 1979; Watts \& Morgan, 1994), especially those who relapse repeatedly in spite of intensive help and who may be challenging or even aggressive in their behaviour. Under such circumstances, clinicians may begin to evoke explanations such as manipulation and deliberate failure to cooperate or even manufacturing of symptoms. We all recognise the need to set limits of behaviour and to establish an effective therapeutic alliance with the patients we treat, but setting limits is probably the most difficult of all clinical skills, and among the most challenging group of patients there are some who are at high suicide risk. They usually divide the opinion of any clinical team almost equally into those who feel that authoritarian measures should be implemented, often leading to discharge, and the remainder who believe that the patient should have a 'further chance'. When such a situation develops, it is useful to review these attitudes in a meeting which involves all members of the team, thereby taking a step back from the heat of confrontation; it is surprising how often a way forward can be agreed once strong feelings have been aired in this way. All this means that effective assessment of suicide risk requires an objective judgement which should include all relevant perceptions of the clinical situation involved.

With the advent of community care, the challenge of suicide risk assessment and management outside the setting of hospital assumes great importance and clear guidelines are even more imperative with regard to clinical procedures. Particularly necessary is an unequivocal policy on what to do when risk escalates, including indices on when to consider the need for hospital in-patient care, which will surely remain necessary when risk is severe. Continuity of care between hospital and community, and a 'care plan approach' which identifies a keyworker as a coordinator and encourages full participation and communication between team 
members, are important principles which have been confirmed by the national Confidential Inquiry into Homicides and Suicides by Mentally Ill People (Royal College of Psychiatrists, 1996).

There is certainly no room for professional rivalry in the assessment and management of suicide risk. The need for extra caution and review of the appropriateness of community care when suicide risk is present is indicated by such factors as:

(a) when the patient is well-known;

(b) when information is incomplete;

(c) after a recent episode of severe deliberate self-harm, especially if suicidal ideation is denied;

(d) when the patient exhibits continuing suicidal ideas, especially if the patient is unable to give reassurance about safety;

(e) when there is gross fluctuation in the degree of distress;

(f) when impulsive behaviour is present;

(g) of there is impairment of insight as a result of psychotic ideation;

(h) if there has been a failure to establish a close empathic therapeutic relationship;

(i) when there is alienation of the patient from others;

(j) when the patient has difficulty in accepting help or maintaining contact with the clinical team;

(k) when there are unresolved adverse life events and situations.

\section{'At risk' supervision registers}

Suicide risk in some degree is so commonly encountered in clinical practice that the role of an 'at risk' register is problematic. The criteria for considering inclusion of patients in such a register need to be precise and clearly agreed by all who are likely to be involved in their clinical care. Such a register may be valuable in the case of patients who tend to relapse into high risk rapidly without giving warning and/or those who tend not to declare their intent clearly. Inclusion should ideally be after full discussion with each patient whose agreement should be sought. Should it be impossible to gain agreement, and inclusion on the register is still regarded as essential, then full explanation for this action should be given, with emphasis on the fact that the process is aimed entirely at reducing risk for the patient, and with reassurance that information is shared only with those who may have clinical responsibility for the patient, for example, on-call staff who may not be familiar with the patient's problems. Clinical situations that fulfil all these criteria are relatively uncommon. It is conceivable that litigation in the case of suicides might focus on whether an 'at risk' register has been used appropriately; full documentation is always a safeguard in such circumstances, as is full agreement between clinicians and managers on agreed guidelines and protocols.

\section{Important clinical subgroups}

\section{Suicidal ideation in the elderly}

Old age is inherently a time of loss in a situation where coping skills may begin to diminish and support structures may be weak. In an isolated individual it is not difficult to imagine loss leading to a feeling of wanting to give up. We often hear older persons say they would like to go to sleep and not wake up, or that they wish they could join their loved ones. This situation may also be complicated by failing physical health or concomitant mental health problems.

There is an important clinical differentiation between an older person who finds it hard to go on and an individual who is actively considering suicide. Skoog et al (1996) studied suicidal feelings in a population sample of 85-year-olds who did not suffer from dementia and found that $4 \%$ had felt that 'life was not worth living' in the previous month. However, only $1 \%$ had though of taking their own life. In the same population, sampling those with mental illness, 29\% felt that life was not worth living, 9\% had thought about taking their own life and $2 \%$ had seriously considered suicide. The relationship between suicidal ideation, deliberate self-harm and subsequent suicide is a complex one, but there is no doubt that the elderly are over-represented both in the statistics of suicide committed after deliberate self-harm and in the suicide statistics more generally (Nowers, 1993, 1997; Hepple \& Quinton, 1997).

Elderly people with suicidal ideas present particular problems of identification and may keep their difficulties to themselves or present their distress in biological terms, often ending up in residential homes and medical wards rather than psychiatric clinics. If the somatisation is recognised, it becomes clear that the elderly are just as capable of being distressed and frightened by suicidal ideas that they may not have felt able to share with others, and equally capable of responding to caring approaches to dealing with them. Old people fear death (particularly that which is self-inflicted) as much as the young. Once face to face and in tune, the clinical techniques in the assessment and management of suicide risk are the same, irrespective of age. 


\section{Suicide encountered in general practice}

In any particular general practice suicide is thankfully rare. What is far more common is suicidal ideation, and many of these patients do not reach the psychiatrist. The 'suicidal ticket' of admission to psychiatric wards is well known and possibly well used, but may be abused. When considering this, it is important to reflect on the fact that psychiatrists formulate risk factors while general practitioners (GPs) have to live with them. The GP has to fulfill the role of combined consultant and senior house officer without the same support in terms of bricks and mortar, additional staff, out-ofhours cover and access to controlled medication.

GPs are no longer afraid to ask about suicidal thoughts. They are usually aware of suicide risk factors and the Health of the Nation targets, as well as the Defeat Depression Campaign, which have all been widely publicised to primary health care teams. The problem the GP has to deal with is the number of patients who admit to having contemplated suicide, and the assessment of severity of risk which each patient presents. GPs measure a degree of risk that may well be different from that recognised by the psychiatrist but they have additional knowledge which is valuable - they know the patient, they know the relative or carers and they know the circumstances. Unfortunately, GPs may be poor at cataloguing all the details in the patient's notes and they do not write a full psychiatric history. This inhibits research when looking at the primary/ secondary interface, but most GPs are accessible by telephone to fill in details which are often valuable in management, if the community mental health team makes the time to contact them.

Management of the suicidal patient in the community setting is a balancing act. Each item on an NHS prescription now costs $£ 5.65$ and weekly prescriptions for the patient who is self-funding are prohibitively expensive. Weekly prescriptions for the elderly, although free, need a level of mobility often not seen. These problems need close liaison with the community pharmacist and community nurse. Dosette boxes filled weekly by the pharmacist and collected by a community nurse, with the pharmacist holding the residual drugs which are prescribed at longer than weekly intervals, is one possible solution. In addition the GP has to deal with confidentiality issues, respect the patient's privacy and often deal with the relatives and carers. GPs need easy access to the community mental health team, with good two-way communication and mutual confidence. In the event of a suicide, the primary health care team is often very traumatised and help and support for the families, reception staff and nurses would often be welcome. GPs may also need help to cope with their own distress and to help them cope with the distress of others.

\section{Young adult males}

These represent the only population subgroup, apart from young Asian females, in which suicide rates have risen in recent years. The precise reasons for this are not clear but we do know that establishing face to face help for young males at risk of suicide can present particular problems. One concerns their reluctance to seek help. A recent study in Avon (Vassilas \& Morgan, 1993) revealed that within the county there was a common picture of rapidly escalating distress in young men who committed suicide, generally in the face of recent upset in the last few days before they died (usually in the setting of confrontation with a partner), who failed to seek outside help. This represents a very great challenge to suicide prevention. Is it their image of themselves or the way in which they perceive helping agencies that leads to their failure to seek help? How can these individuals best be reached? Should we develop pro-active strategies, and if so, where are the best points of contact? It may well be that approaches may need to vary from one community to another, whether rural, urban or inner-city. The increased use of carbon monoxide from car exhaust fumes as a means of suicide by young men emphasises the importance of strategies such as fitting catalytic converters to cars, and even more simply changing the design of car exhaust pipes. Alcohol and substance misuse are also key issues in young male suicides. Some relevant risk factors are listed in Table 1.

\section{Major psychosis}

Affective psychosis carries the highest level of suicide risk. The proportion of people who commit suicide who suffer from a schizophrenic illness is increasing and it is probably now in the region of $10 \%$ of all suicides. The clinical risk factors for suicide in schizophrenic illness are set out in Table 1. In trying to explain the increase in suicides in people with schizophrenia we also need to look closely at the social factors involved, with particular focus on adverse living conditions. Does community care meet the needs of people with schizophrenia? Can we feel reassured that young people with schizophrenia in our own service receive adequate continuity of care, rather than moving anonymously from one setting to another? When they need 
hospitalisation, do they receive it close to their homes rather than at long distances away, just as they did in the old Victorian-style mental hospital service? If we cannot answer these questions in a satisfactory way, then it should not be a surprise that suicide rates in people with schizophrenia are increasing.

\section{Personality disorder}

This diagnostic category is often linked closely with alcohol and substance misuse, itself associated with high risk for suicide.

The Confidential Inquiry (Royal College of Psychiatrists, 1996) comments that clinicians tend not to exploit fully the value of Mental Health Act procedures in the management of suicide risk. This may apply particularly to the care of those with personality disorder. It may be insufficiently acknowledged that personality disorder can at times be punctuated by relatively short-lasting crises, often depressive in nature and which may involve serious suicide risk. Setting limits of behaviour in patients with personality disorder, stressing individuals' responsibility and capacity with regard to their own risk management, is an essential clinical strategy. Only in this way is it possible to counter the risk of malignant overdependence, often related to regression, particularly in the case of patients with borderline personality disorders. Yet it can be difficult to alter the direction of our approach to consider the possibility that judgement may have become grossly impaired, perhaps to such an extent that compulsory inpatient assessment or treatment might reasonably be considered. A reluctance to enter into the compulsory admission situation concerning a person who has exhibited long-standing behavioural disorder is, of course, understandable, because it can then be difficult to know exactly when to end the order. Nevertheless, such individuals occasionally commit suicide and these very difficult clinical issues need to be confronted if we are to be effective in preventing them. A full multidisciplinary approach through a care plan is helpful in facing up to this particular clinical dilemma, particularly in deciding when such a compulsory order should be terminated once the specific crisis of suicide risk is over.

\section{References}

Bannerjee, S., Bingley, W. \& Murphy, E. (1995) Deaths of Detained Patients: A Review of Report to the Mental Health Act Commission. London: Mental Health Foundation.
Hepple, J. \& Quinton, C. (1997) One hundred cases of attempted suicide in the elderly. British Journal of Psychiatry, $171,42-46$.

Morgan, H. G. (1979) Death Wishes? The Assessment and Management of Deliberate Self-Harm. Chichester: Wiley.

— \& Priest, P (1991) Suicide and other unexpected deaths amongst psychiatric in-patients. The Bristol confidential inquiry. British Journal of Psychiatry, 158, 368-374.

— \& Stanton, R. (1997) Suicide among psychiatric in-patients in a changing clinical scene. Suicidal ideation as a paramount index of short-term risk. British Journal of Psychiatry, 171, 561-563.

Nowers, M. (1993) Deliberate self-harm in the elderly. A survey of one London borough. International Journal of Geriatric Psychiatry, 8, 609-614.

- (1997) Suicide and deliberate self-harm in the elderly. Current Opinion in Psychiatry, 10, 313-317.

Royal College of Psychiatrists (1996) Report of the Confidential Inquiry into Homicides and Suicides by Mentally Ill People. London: Royal College of Psychiatrists.

Skoog, I.,Aevarsson, O., Beskow, J., et al (1996) Suicidal feelings in a population sample of non demented 85-year-olds. American Journal of Psychiatry, 153, 1015-1020.

Vassilas, C. A. \& Morgan, H. G. (1993) General practitioners' contact with victims of suicide. British Medical Journal, 307, 300-301.

Watts, D. \& Morgan, H. G. (1994) Malignant alienation. British Journal of Psychiatry, 164, 11-15.

Williams, R. J. \& Morgan, H. G. (eds) (1996) Suicide Prevention. The Challenged Confronted. NHS Advisory Service Thematic Review. London: HMSO.

\section{Multiple choice questions}

1. People at risk of committing suicide:

a exhibit an unchanging level of distress

b do not talk of suicidal impulses

c often seek help in the last weeks before their death

d may present a concomitant risk of violence to others

e can become seriously alienated from others.

2. Recent trends in suicide rates indicate:

a a rapid rise in suicide among young men

b a fall in suicide among young Asian women

c a rapid rise in suicide rates in young women in general

d falling rates of suicide associated with schizophrenic illness

e that suicide is more common in older rather than younger men.

3. Features which may mislead in the management of suicide risk include:

a gross variability of manifest distress

b denial of suicidal ideas

c misleading (short-lasting) clinical improvement on admission to hospital

d absence of distress in some with serious suicidal intent

e angry provocative behaviour. 
4. Psychiatric in-patients at risk of suicide:

a often commit suicide when on leave from the ward

b are at reduced risk immediately after admission

c symptomatic improvement is a sufficient index to indicate that discharge can be safe

d can provoke conflict between clinical team members

e usually exclude depressive symptoms as their main clinical feature.

5. Risk factors for suicide:

a are highly specific and sensitive

b are more effective in predicting suicide in the long-term than in the immediate future

c reflect a distinctive clinical stereotype of suicide d ideally should be used to back-up direct face to face clinical assessment

e should accord paramount importance to regular evaluation of suicidal ideation.

\begin{tabular}{|llllllllll|}
\hline MCQ answers & & & & & & \\
1 & & 2 & & 3 & & 4 & & 5 & \\
a & F & a & T & a & T & a & T & a & F \\
b & F & b & F & b & T & b & F & b & T \\
c & T & c & F & c & T & c & F & c & F \\
d & T & d & F & d & T & d & T & d & T \\
e & T & e & F & e & T & e & F & e & T \\
\hline
\end{tabular}

\section{Using the Mental Health Act A Training Resource for Doctors}

Prepared by the Royal College of Psychiatrists' Working Group

A good knowledge of the Mental Health Act 1983 is vital for psychiatrists to function effectively in today's mental health services, particularly since the use of compulsory admission to hospital has risen considerably over recent years. The Act also has important implications for care outside hospital. General practitioners are regularly involved in using the Act, and need to be aware of its provisions.

This training pack is intended to support the development of better training for psychiatrists seeking approval under Section 12 of the Act and to support the continuing education of psychiatrists and GPs. It comprises a 45 minute video, comprehensive written guidelines and lecture notes, together with overhead projector masters. It is intended as an aid (a) to those running training seminars within hospitals or trusts and others wishing to set up their own seminars, and (b) to individual practitioners who work in more isolated settings and who may wish to use distance learning.

Published 1997, ISBN1 901242 099 9, 93 page text +18 unbound presentation masters, 1 PAL video cassette 45 min length. Video cassette and text held together in a white PVC ring binder. Price $£ 45.00+$ VAT.

Available from Book Sales, Publications Department, Royal College of Psychiatrists, 17 Belgrave Square, London SW1X 8PG. Credit card orders can be taken by telephone

(Tel. +44(0)171 235 2351, extension 146). 